\title{
Proposal for the Conceptual Design of Aeronautical Final Assembly Lines Based on the Industrial Digital Mock-Up Concept
}

\author{
Fernando Mas ${ }^{1}$, Alejandro Gómez ${ }^{2}$, José Luis Menéndez ${ }^{1}$, and José Ríos ${ }^{2}$ \\ ${ }^{1}$ AIRBUS, Av. García Morato s/n, 41011, Sevilla, Spain \\ fernando.mas@airbus.com \\ ${ }^{2}$ Universidad Politécnica de Madrid, José Gutiérrez Abascal 2, 28006 Madrid, Spain \\ jose.rios@upm.es
}

\begin{abstract}
The design of a Final Assembly Line (FAL) is carry out in the product industrialization activity. The phase dealing with the definition of conceptual solutions is characterized by depending heavily on the personnel experience and being time-consuming. To enhance such process, it is proposed a development of a knowledge based software application to assist designers in the definition of scenarios and to generate conceptual FAL alternatives. Both the scenario and the generated FAL solution are part of the industrialization digital mock-up (IDMU). A commercial software application used in the aircraft programmes and supporting the IDMU concepts of: Product, Process and Resource; was selected to implement a software prototype. This communication presents the adopted methodological approach and the architecture of the developed application.
\end{abstract}

Keywords: aeronautical final assembly line conceptual design, industrial Digital Mock-Up, PLM systems.

\section{Introduction}

The design of a Final Assembly Line (FAL) can be decomposed into three assembly line design phases: concept, definition and development. The FAL design is part of the product industrialization activity. Such industrialization activity can be decomposed into three main sub-activities: "create conceptual assembly process", "define assembly process" and "develop detailed assembly process". Each of these activities is carried out in its corresponding assembly line design phases: concept, definition, and development [1].

Requirements are under definition during the conceptual design phase of an aeronautical FAL. For instance: work share, work load distribution, factory locations, production processes and technologies, main machinery and tooling. During the conceptual phase, designers require defining FAL alternatives with different values for the input requirements. A set of values for the input requirements defines a scenario and allows executing 'what-if' analyses [2]. A Project Management Office (PMO) is responsible to define the scenarios. 


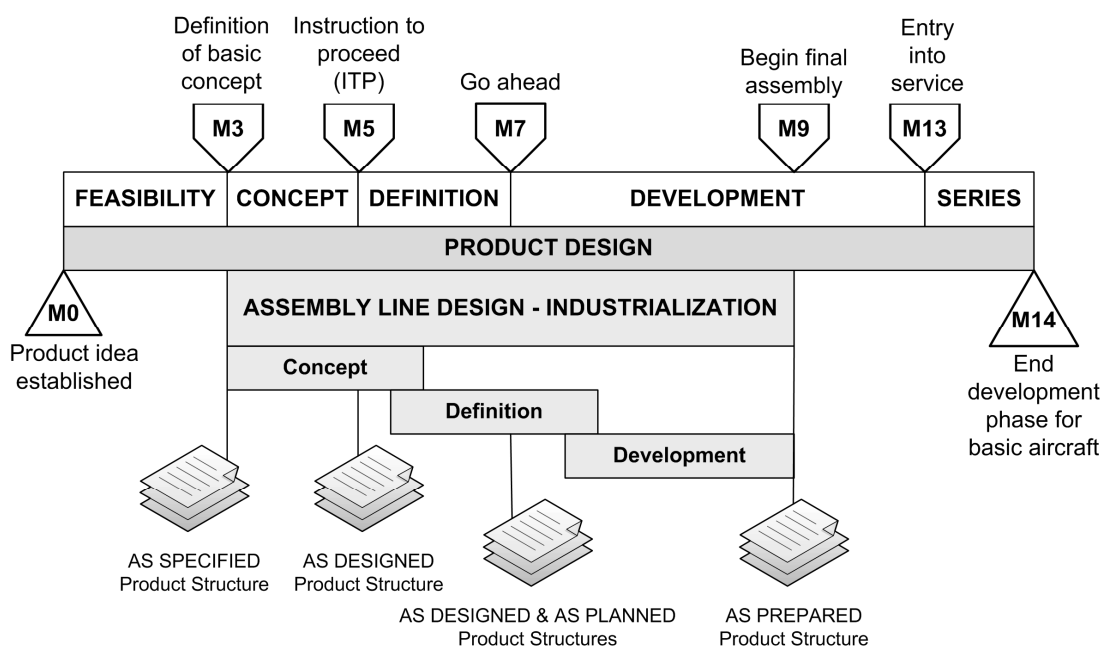

Fig. 1. AIRBUS Product lifecycle and development milestones

The design of the whole plane, and its corresponding subassemblies, evolves along the design process, four types of product definition structures are created along the lifecycle: 'as specified' (M3), 'as designed' (M5), 'as designed \& as planned' (M7), and 'as prepared' (M9) [3]. The design of a FAL is shifted from the product lifecycle, starts in M3 and ends in M9 (Fig. 1). The conceptual functional aircraft definition, mainly the product configurations 'as design' and 'as planned', is responsibility of the Design Engineering (DE) in collaboration with the Manufacturing Engineering (ME). Based on the product configuration and the scenario, the ME is responsible to execute the case and to define the digital mock up of the industrialization solutions or FAL alternatives. As a result, the 'as prepared' product structure is created. The final decision is responsibility of the PMO. Both the scenario and the FAL design are part of the Industrialization Digital Mock-Up (IDMU). An IDMU comprises product, processes and resources information, both geometrical and technological [4]. PLM systems are the tool to create, manage and support any digital mock-up related to products, processes and resources [4-6] (Fig. 2).

At the conceptual phase, the process of generating industrialization solutions depends heavily on the personnel experience and it is time-consuming. Consequently, manufacturing engineers can only check a simplified set of cases to generate and submit early manufacturing processes and resource requirements. In order to enhance such process, it was decided to investigate the development of a software application to assist designers in the definition of scenarios and to generate FAL alternatives at the conceptual stage. The development had to be carried out within the framework of a commercial PLM/CAX system used in the aircraft programs (Catia/Delmia v5) (Fig. 2). 


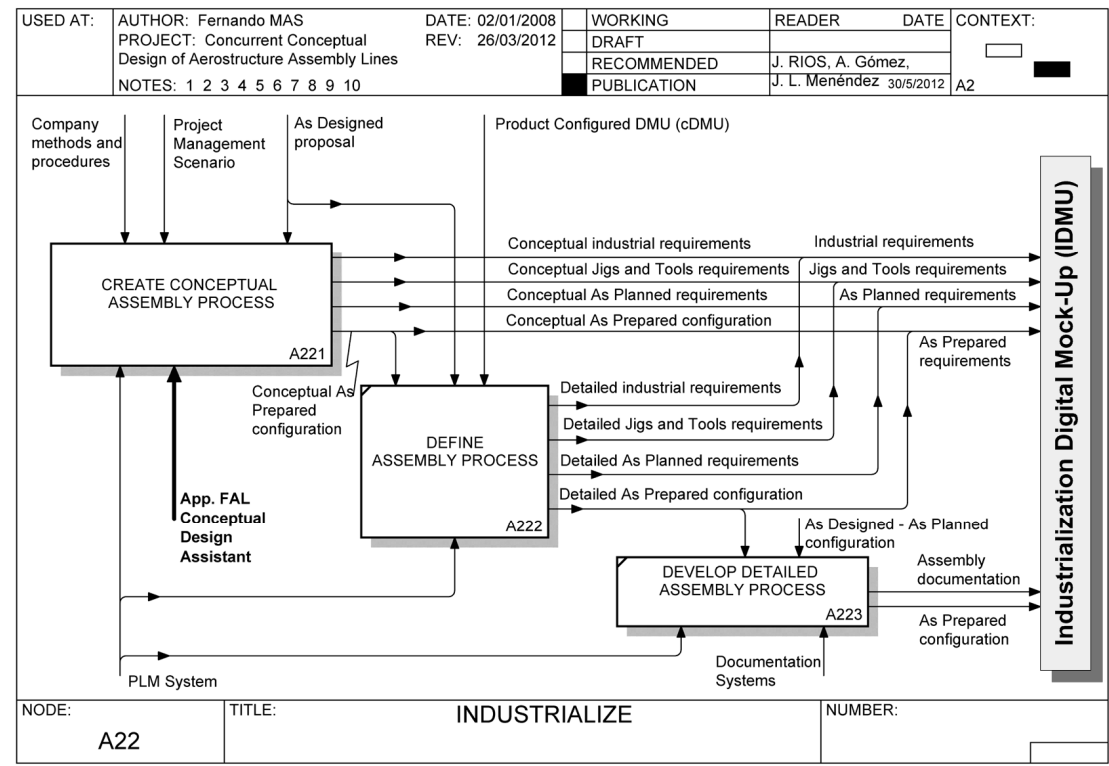

Fig. 2. IDEF0 Context diagram for the Application FAL Conceptual Design Assistant

This communication presents the methodological approach followed along the development process and the architecture of the developed application. In the next section, the methodological approach adopted in the work and the models, created to support the development of the application, are briefly presented.

\section{Methodological Approach and Developed Models}

Rather than relying on experience and rules of thumb, the literature shows the need to develop formal models of manufacturing systems for analysis and decision making [7-8]. The literature shows also that the development of aid tools, to assist in the design of process and aircraft assembly lines, is a topic of interest. An example is the development of an aid tool, based on the definition of an assembly graph, which allows the specification of several alternatives for processes and factory layouts, in a way that they can be compared within a CAD environment and transferred into a simulation software tool for task scheduling analysis [9].

The development of a software application, to assist designers in the definition of scenarios and to generate FAL alternatives at the conceptual stage could not be identified in the literature. Such development requires capturing and reusing product and process knowledge in an integrated way. IDEF0 (Integrated DEFinition for function modeling) and UML (Unified Modeling Language) were selected to define the methodological approach adopted in this work and the resulting models. 
The first task was a literature review to analyze both the product design process on engineering design and the assembly line design process described in textbooks. While most textbooks on engineering design provide a similar general structure for the design process with three main phases: Conceptual Design, System level or Embodiment Design and Detail Design; textbooks on assembly line design rather than providing a systematic process, provide a general view of the elements involved in the assembly line design and tend to focus on the assembly line balancing task [1]. The second review task was the creation of an 'as is' IDEF0 process model based on the industrial practices from previous aircraft projects. From the analysis of such 'as is' model two main elements were identified: process improvements actions and activities that could benefit from the development of software utilities [4]. Based on these two inputs, a 'to be' IDEF0 process model focused on the Industrialize activity was defined. The process model shows the activities to conduct, the flow of information and helps to identify the concepts and knowledge involved in the aircraft FAL conceptual design process $[1,10]$.

The next step was to develop a knowledge model using UML. The knowledge modeling of aircraft assembly lines requires reviewing works dealing with modeling of assembly information, processes and lines [1]. From such review was concluded that the semantic concepts involved in the conceptual design phase of an aircraft assembly line were not fully taken into account in the identified models. Models presented in the literature provide three main views: product, process and line balancing. The modeling of the conceptual phase demanded to integrate and to extend concepts from the three views, particularly from the process view. The proposed conceptual model was divided into three interrelated sections or knowledge units: Product, Processes and Resources; that together constitute the IDMU [1]. The product section comprises the concepts to define the joints to be assembled and, both the functional (as designed) and the industrial (as planned and as prepared) views. The process section comprises the concepts, in terms of technology, sequencing and resources, to define a procedure to assemble each joint defined in the product section. Technology, sequencing and resources are collected in the work station concept, and work stations are grouped into the assembly line concept. The resources section comprises the concepts to define three main types of resources: jigs and tools, industrial means and human resources.

To implement the developed IDMU model, classes were mapped into elements of the commercial software (Catia/Delmia v5), where the IDMU is created. Catia/Delmia v5 provides the PPR structure (Process-Product-Resource) to support the IDMU concept. The model is implemented by means of Catia v5 macros in VBA language that use the Catia v5 Application Programming Interface (API). A process document (.CATProcess) integrates three lists corresponding to: Product, Process and Resource. A component is the basic class in the product knowledge unit (KU). A component is defined in a file of type CATPart and corresponds to the lowest level in a product tree. There are three product trees to represent the three product views: as designed, as planned and as prepared. Each product tree has its corresponding nodes: 
functional_nodes, industrial_nodes and as_prepared_nodes. The classes AsPlanned and AsPrepared were defined to generate the corresponding product structures. Each product structure is defined in a file of type CATProduct. The nodes of a product tree can point to a CATProduct file (intermediate elements) or to a CATPart file (lowest level individual element). The joint class is linked to a CATProduct file. The joint class has a link to the Basic_assembly_process class. An object of the Basic_assembly_process class is defined in a CATProcess file. Fig. 3 shows the mapping of the proposed model into classes defined in CATIA (shadowed) and the corresponding type of files where instances are stored.

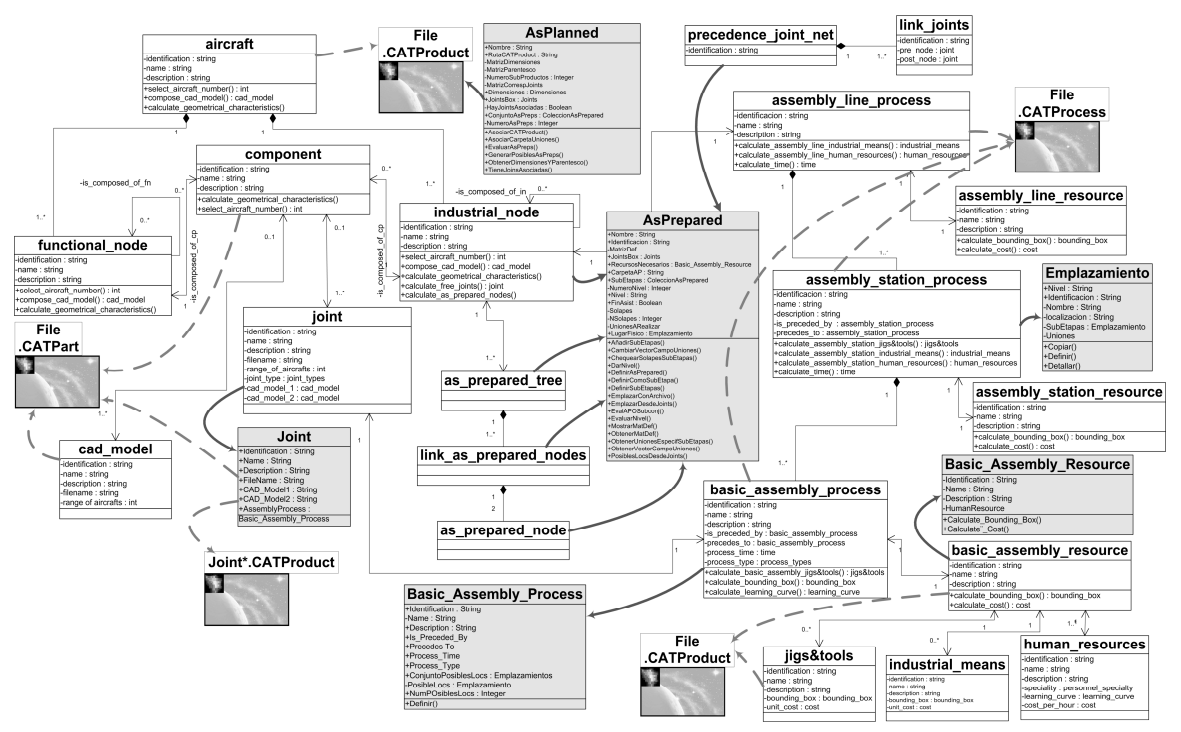

Fig. 3. Mapping of the developed UML class model into Catia v5 concepts

\section{Results: FAL Conceptual Design Assistant Tool Prototype}

The main result is an assistant tool, integrated within Catia v5, which helps designers to generate FAL alternatives by defining scenarios. The application generates technological information integrated within an IDMU supported by the commercial PLM system. The current development implements the three main elements: product, processes and resources. A very simple aircraft model was created and used to test the application. The results obtained in the executed case studies relate to requirements for: space, transport, resources, industrial means and cost; and allow validating the conceptual approach adopted in the research. Fig. 4 shows the main tasks supported by the assistant tool prototype, such tasks are executed within the activity 'Create Conceptual Assembly Process' A221 (see Fig. 2). 


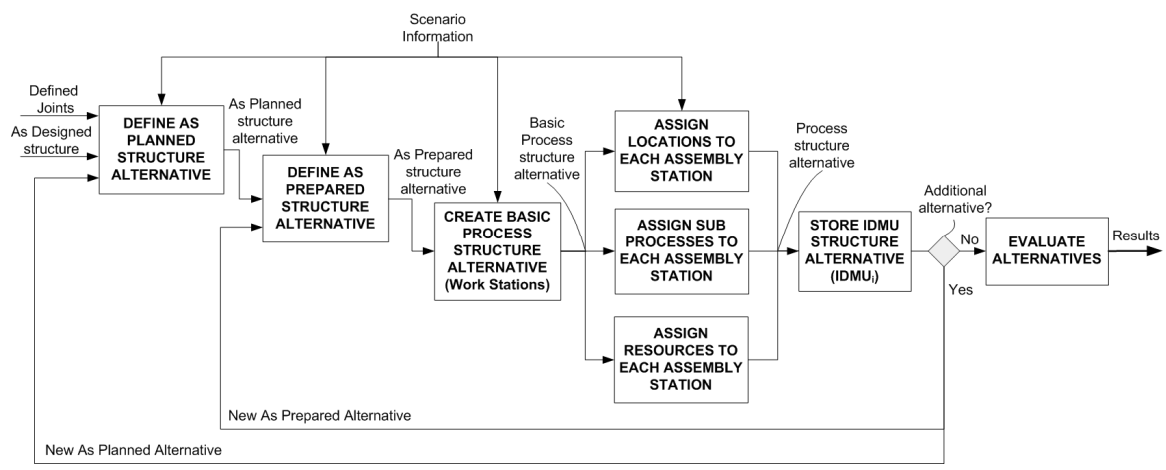

Fig. 4. Architecture of the FAL conceptual design assistant tool prototype

Defining an assembly process alternative, as proposed in the assistant application (Fig. 4), requires using the scenario information and involves fixing an assembly sequence, establishing sub-assemblies associated to the sub-stages of the process, locating them into real industrial plants belonging to the set of available company's facilities, adding sub processes depending on the type of joint to be executed (e.g.: fuselage join-up) and assigning the resources to be used (e.g.: NC machine, overhead crane).

An assembly sequence comprises defining the order of execution of the required 'Joints'. Such ordering provides a hierarchical precedence structure with possible parallel branches; that is the 'As Prepared' product model view. Along the execution of the application, the user defines the sequence by selecting the different joints in inverse order of execution from last to first. After selecting each joint, the user can select its predecessors.

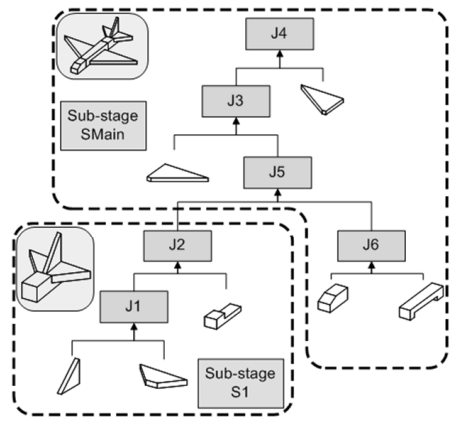

Alternative A1

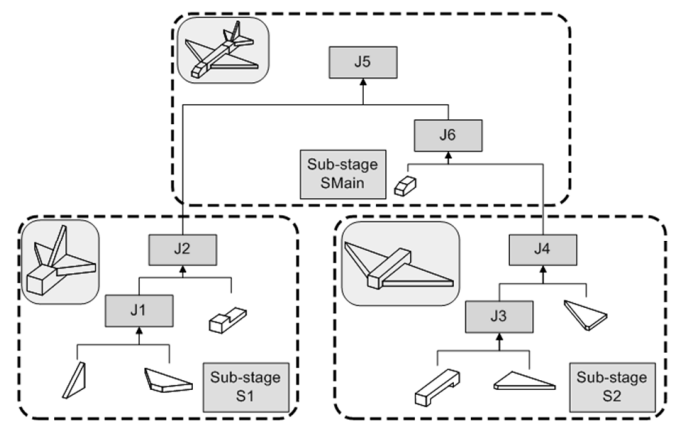

Alternative A2

Fig. 5. Example of two alternatives for the 'as planned' and 'as prepared' structures

Once the sequence is defined, sub-stages must be defined. Each one must contain a number of executions of joints and is related with a sub-assembly or set of components depending on the position of the involved joints within the sequence. Each sub-stage is an industrial node. Fig.5 shows two examples of assembly process alternatives. Alternative A1 comprises two sub-stages, called S1 and SMain. Joints J1 and 
$\mathrm{J} 2$ are executed at S1, and the rest of the joints, at SMain. S1 and SMain will be located in different industrial plants. Locating sub-stages is controlled by a constraint: the user can select exclusively industrial plants where the assembly processes involved in the sub-stage can be made (scenario information). Each joint object is related with an instance of class BasicAssemblyProcess (Fig. 3), which has an attribute related to the possible plants where can be carried out. In the example of Fig. 5, alternative A1, substage S1 can only be located in those plants where joints $\mathrm{J} 1$ and $\mathrm{J} 2$ (actually their associated assembly processes) can be executed. Feasible locations for each sub-stage are shown to the user in this step of the assistant application execution. A detailed discussion of a case study, executed with a prior version of the assistant tool, where the product knowledge unit was implemented, is presented in Gómez et al. [11].

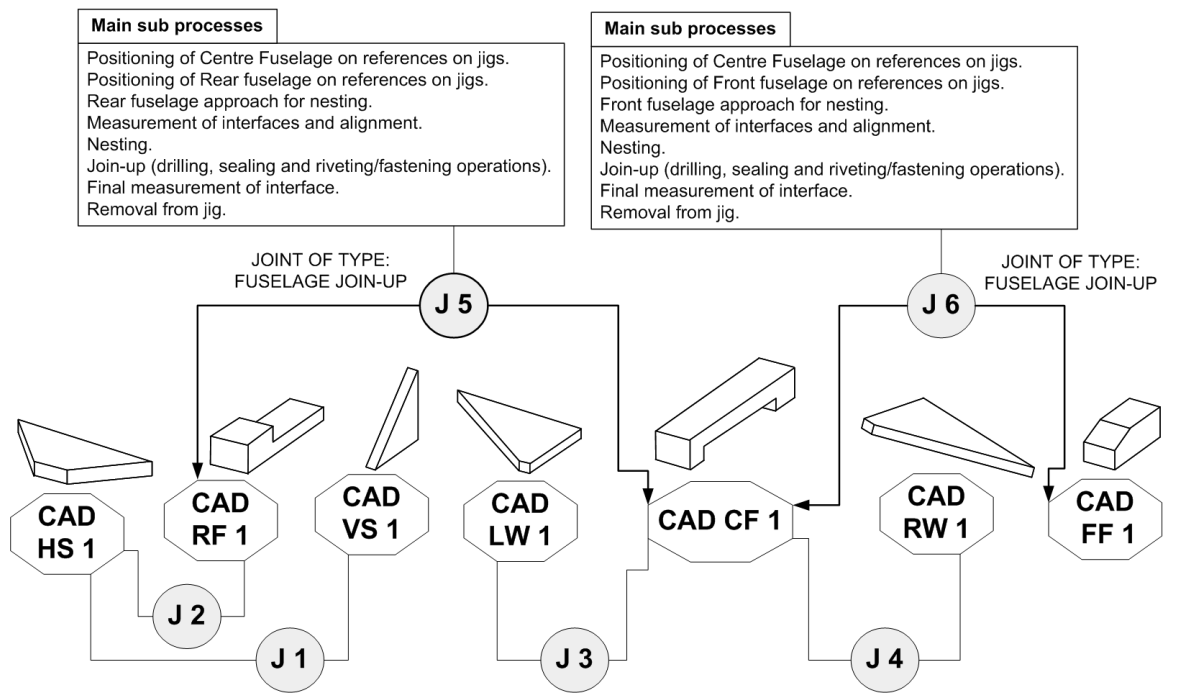

Fig. 6. Main sub processes to be carried out to execute a joint of type Fuselage Join-Up

The next step is to assign sub processes to the work stations. A library with a set of basic types of joints was defined, where each basic type comprises the main sub processes to be carried out. Fig. 6 shows the example of the Fuselage Join-Up joint type. For each work station, depending on the type of joints to be executed in it, the design assistant, making use of the joint sub process library, generates automatically the main level of sub processes to be carried out and the corresponding nodes are created in the Process List structure provided by Catia/Delmia v5.

The last step in the configuration of the process structure alternative is the assignment of resources. The assistant tool provides a list of possible resources to the designer. Each resource has a set of basic attributes for its basic definition (e.g.: bounding_box, capacity, cost). The designer has to select the resource type, input the value for each attribute and select the process node where the resource will be used. The assistant tool will generate a node in the Resources List structure provided 
by Catia/Delmia v5. The geometric definition of the resource is out of scope of this work and it will have to be created separately.

At this stage, a conceptual structure of a possible FAL solution is defined. Since an 'as designed' product structure may have more than one 'as planned' alternative. The 'as planned' structure is defined by grouping components into different subassemblies which represent industrial nodes in the real process. And an 'as planned' structure may have more than one 'as prepared' structure. The 'as prepared' structure is created by sequencing the execution of the joints to get the sub-assemblies defined at the 'as planned' structure. Once the first possible process structure (i.e.: FAL alternative) is created, the designer is requested to select if more alternatives need to be evaluated.

The evaluation of FAL alternatives defined at the conceptual phase relates to estimate: space requirements, transportation requirements, cost, and operation time. Space requirements for a specific assembly plant are bigger when a higher number of joints must be executed in it, due to the increase on the number of components to be processed. Transport requirements depend on the distance between the chosen locations and the dimensions of the intermediate products to be transported. The whole assembly process cost and operation time is strongly related with the resources to be used and the transport requirements, the space utilization has also a high impact in the final cost. In the last part of the assistant, each FAL alternative is evaluated according to the five criteria: dimensions, resources requirements, transport requirements, time and cost. The coding needed to implement the resources requirements evaluation is currently in progress.

To execute the evaluation of alternatives, three main generic utilities were developed: the tree structure analyzer, the component dimensions calculator, and the node properties calculator. The product tree analyzer captures the Components Tree Structure of a CATProduct file. The function was programmed with a recursive algorithm which is valid for any product structure. This utility allows capturing information about the product model view structures, which are represented by CATProducts files. This utility could be adapted to analyze a resource structure.

The component dimensions calculator determines the dimensions of a product. Dimensions of each component are calculated by defining parallel planes and checking if the part is cut by them. If that condition is false, the distance between planes is reduced. The loop is executed until precision required is achieved. Dimensions of an assembly are calculated by using the dimensions, the relative positions and the orientations of each of its components. This utility is linked with the industrial facilities space requirements and the space evaluation for transportation.

The node properties calculator allows finding a property in the components or defining it if it is not found. It also calculates the value of the property in all the nodes of the product tree by using the utility to capture its structure and applying an adding upward algorithm. This utility is used to calculate the cost at each node of the product tree. Fig. 7 shows the assistant user interface when linking a resource to a process node, and the PPR trees created by the assistant when executing one of the cases showed in Fig. 5. 


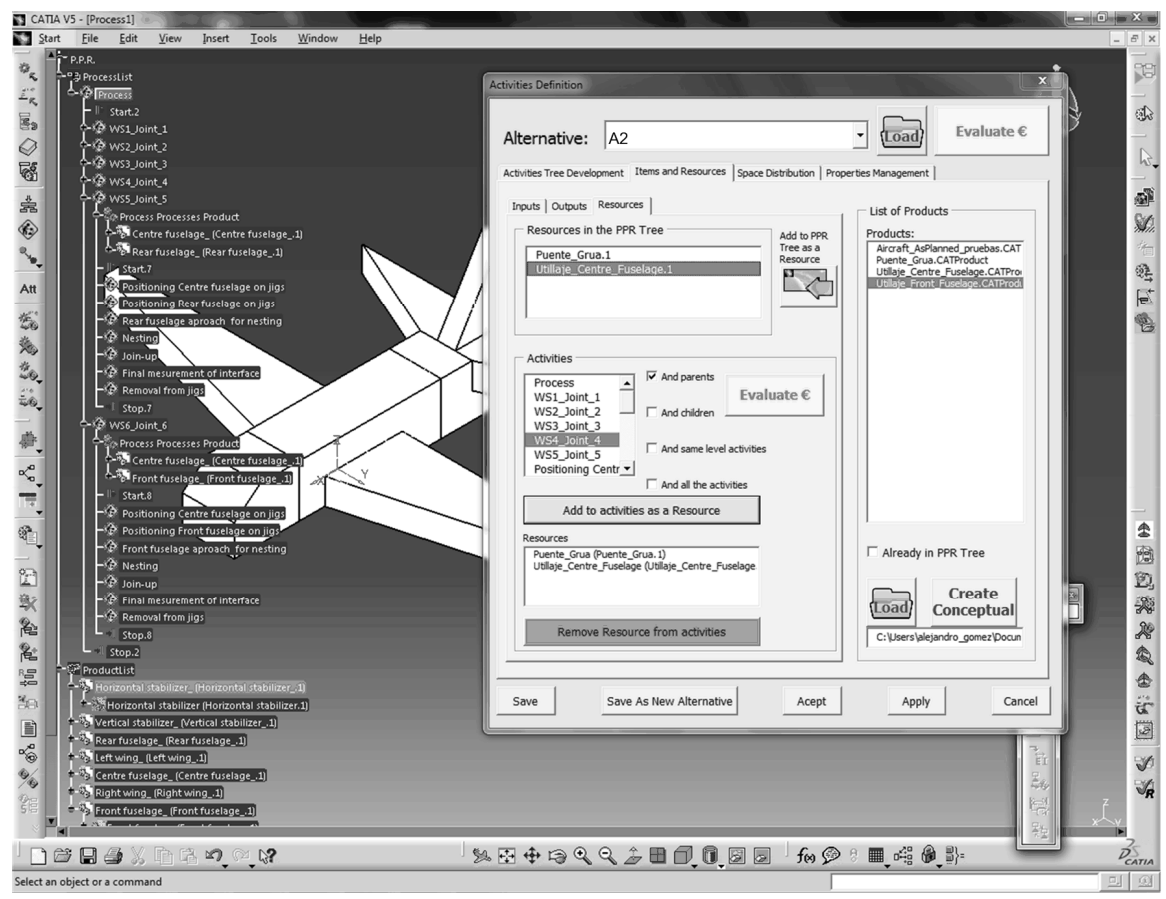

Fig. 7. Assistant user interface example: activity definition window and resource linking

\section{Conclusions}

This paper presents a proposal to assist designers during the conceptual design of an aircraft final assembly line. The approach is based on the definition of an Industrial Digital Mock-Up (IDMU). To create the IDMU an ad-hoc assistant tool was modelled, developed and integrated within a commercial software system (Catia/Delmia v5). The assistant tool allows defining different alternatives and evaluating them in terms of: dimensions, resources requirements, transport requirements, time and cost. Although, the resources requirements evaluation is currently under development, the current prototype confirmed the feasibility of the approach. The reader is referred to Gómez et al. [11] for a detailed discussion of a case study executed with a prior version of the assistant tool, where the product knowledge unit was implemented.

Although the evaluation of the alternatives is conducted at the industrialization conceptual phase, when products are still preliminarily defined, the evaluation of different scenarios allows creating estimates that help designers during the decision making process.

In addition to the already mentioned fully implementation of the resource knowledge model, future work aims an automatic process planning capability in the form of an algorithm to create the 'as prepared' alternatives from the information defined in the 'as planned' structure, the joints to execute and the assembly process information.

Acknowledgments. The authors would like to thank the AIRBUS Military colleagues for their contribution during the development of this work. 


\section{References}

1. Mas, F., Ríos, J., Menéndez, J.L., Gómez, A.: A process-oriented approach to modeling the conceptual design of aircraft assembly lines. Intl. J. of Advanced Mfg. Technology 62 (2012)

2. Mas, F., Ríos, J., Menéndez, J.L.: Scenario for concurrent conceptual assembly line design: a case study. In: AIP Conf. Proc., 4th Mfg. Eng. Soc. Intl. Conf., MESIC 2011, vol. 1431, pp. 625-632 (2011)

3. Pardessus, T.: Concurrent engineering development and practices for aircraft design at Airbus. In: Proc. of the 24th ICAS Conf., Yokohama, Japan (2004)

4. Menéndez, J.L., Mas, F., Serván, J., Ríos, J.: Virtual Verification of an Aircraft Final Assembly Line Industrialization: An industrial case. Key Engineering Materials 502, 139 144 (2012)

5. Delpiano, M., Fabbri, M., Garda, C., Valfré, E.: Virtual Development and Integration of Advanced Aerospace Systems: Alenia Aeronautics Experience. RTO AVT Symposium, Paris, RTO-MP-089 (2002)

6. Van Wijk, D., et al.: Integrated Design and PLM Applications in Aeronautics Product Development. In: Proc. of the 19th CIRP Design Conf. - Competitive Design, Cranfield University, pp. 128-133 (2009)

7. Fowler, J.W., Rose, O.: Grand Challenges in Modeling and Simulation of Complex Manufacturing Systems. Simulation 80, 469-476 (2004)

8. Butterfield, J., Crosby, S., Curran, R., Price, M., Armstrong, C.G., Raghunathan, S., McAleenan, D., Gibson, C.: Optimization of aircraft fuselage assembly process using digital manufacturing. J. of Computing Information Science Engineering 7, 269-275 (2007)

9. Anselmetti, B., Fricero, B.: Aid tool for the design of process and aircraft assembly lines. Aerospace Science and Technology 23, 387-398 (2012)

10. Mas, F., Ríos, J., Menéndez, J.L., Hernández, J.C., Vizán, A.: Concurrent conceptual design of aero-structure assembly lines. In: Proceedings of the 14th Intl. Conf. on Concurrent Enterprising (ICE), Lisbon, Portugal, pp. 783-790 (2008)

11. Gómez, A., Mas, F., Menéndez, J.L., Ríos, J.: Development to assist in the creation of product development structures. In: Proc. of the 29th Intl. Manufacturing Conference (IMC29), Belfast, UK (August 2012) 